\title{
Nutrition and Immune Function
}

\author{
Neharika Saxena $^{* 1}$, H M Saxena ${ }^{2}$ \\ ${ }^{I}$ Department of Veterinary Public Health, College of Veterinary and Animal Science, \\ RAJUVAS, Bikaner, Rajasthan 334001. \\ ${ }^{2}$ Department of Veterinary Microbiology, COVS, GADVASU, Ludhiana 141004
}

\begin{abstract}
Nutrition is significant modulator of the resistant function and can often tip the balance between health and syndrome. Many types of syndromes are due to malnutrition. Specific concentrations of nutrients are required for optimal health of the animal. The diet supplies energy and amino acids that contribute to the development, maintenance and use of the immune system. Substrates are necessary for the anabolic activity of immune system's cells (leukocytes), such as proliferation and antibody production as well as the secretion by the liver of large quantities. In young animals, a rigorous deficiency of any nutrient impairs immunocompetence. Nutrients can affect the functions of leukocytes and hence the type, duration, and magnitude of the immune response. It is important to determine the quantitative nutrient requirements of the immune system for its development in the young animal, its maintenance at times of good health, and its poor immunocompetence, in turn, can result in greater incidence and duration of infections, leading to decreased food intake, nutrient losses, and impaired animal health and well-being. There are two basic causes of degenerative diseases: immune dysfunction due to toxicity, and nutritional deficiency. Deficiency arises from poor soil and depletion of nutrition in processed feeds. Many types of illnesses and diseases are caused by malnutrition. Target animal species require to be examining to decide specific concentrations of nutrients for best immunocompetence and physical condition.
\end{abstract}

Keywords - Immunocompetence; Syndromes; Nutrients

\section{Introduction}

\subsection{Nutrients and their Roles in Immunophysiology and Homeostasis}

Water acts as a solvent for a mixture of biochemical reactions. It is needed to maintain vascular volume, for supplying nutrients to tissues, and to remove waste from the body. Deficiency of water impairs homeostasis and body functions and adversely affects the health. Body requirement of water varies with physical activity and environmental conditions.

Energy vital for sustaining diverse functions of the body, consist of respiration, physical work, circulation and protein synthesis is given by carbohydrates, proteins and fats in the fast. Carbohydrates (sugars and starches) provide energy to cells in the body. Fibre has an important role in gut health and has its nutritional value.

Dietary Fiber is non-digestible carbohydrates and lignin that are inherent and unharmed in plants. Sticky fibers hindrance the gastric emptying of ingested foodstuffs into the small intestine, which can effect in a sense of fullness. This delayed emptying effect also results in reduced blood glucose concentrations. Fibers can also interfere with the absorption of dietary fat and cholesterol, and the recirculation of cholesterol and bile acids in the liver and intestine. The overuse of fibre has negative effects because the animal cannot digest it all and it leaves less space for materials like protein and amino acids. Too little fibre, on the other hand, limits gut stimulation and can contribute to gut diseases such as colitis and ileitis. Acute constipation problems may occur with too little crude fibre in the diet. One of the most common groups of fibres, the non-starch polysaccharide (NSP) fibres, is beneficial to gut health at certain levels, but not at higher levels. At high levels of NSP, protein retention is slowed because nitrogenous compounds are bound by the fibre in the small intestine and transported to the hind gut without being digested. Medium to high levels of NSPs also provide an excellent substrate for anaerobic fermentation in the hind gut leading to acute diarrhoea via a disturbed electrolyte balance and overgrowth Low levels of NSPs, however, are fine and can promote a useful effect where gram positive bacteria, such as the lactobacillus colonize the gut wall to provide gut protection.

Polysaccharides help the immune system to destroy cancer cells and viruses. However, each type of polysaccharide has its own unique ability and healing effect on certain cancers. Proteins form the major structural components of all the cells of the body. All along with amino acids, they role as enzymes, hormones and membrane carriers,

Amino acids are the building blocks of protein. Nine amino acids are considered essential and thus must be provided through the diet. The relative ratio of essential amino acids in a food protein and its digestibility determines the quality of the protein. Threonine is a high proportion of the cellular components of the gut wall and when the animal is in a poor state of health or when a fibre- 
rich diet is fed, endogenous losses are high and additional threonine will be required in the diet.

Tryptophan is a precursor of the polypeptide serotonin that is involved in feed intake regulation and also in behavioral expression. In case of infections with some bacterial serotypes - leading to acute lung inflammation, plasma tryptophan levels fall significantly in contrast to the other essential amino acids.

Fats provide energy to the body and help in the absorption of fat-soluble vitamins, carotenoids etc. Unsaturated fatty acids in the rumen have a detrimental effect on rumen micro-organisms. This results in decreased fibre digestibility and thereby a reduced dry matter intake. Especially during the transition phase and in early lactation cows are already in a negative energy balance. Maximizing dry matter intake is crucial for animal health and production. Pregnancy rates can increase $15 \%$ when fatty acid nutrition is optimized for fertility. Vitamins are present in plants and are necessary for health. They work synergistically with other vitamins and some nutrients.

Phytochemicals are grouped into four broad categories, namely indoles, isothiocyanates, flavonoids and isoflavones. While some plants are rich sources of a particular phytochemical, others contain myriads of phytochemicals. Every plant also contains its own unique variety of phytochemicals. For example, indoles, one of the larger groups of phytochemicals, is available in vegetables. Flavonoids have anti-cancer properties and prevent cancer causing hormones from attaching to normal cells. They also inhibit enzymes responsible for cancer cell metastasis. Free radicals are produced by toxic chemicals, sunlight and metabolic processes in the body. They can damage proteins, membranes and DNA, leading to cancer and other degenerative diseases. Antioxidants obtained from natural plant sources seek out and "anti-oxidize" free radicals to prevent their destructive effect on the body.

Trace elements exist in cells and tissues of the animal body in a variety of chemical combinations, and in characteristic concentrations. The concentrations of trace minerals must usually be maintained within quite narrow limits if the functional and structural integrity of the tissue is to be maintained alongside the expansion, health, and productivity. Ingestion of diets deficient, imbalanced, or excessively high in trace minerals may induce changes within the form or concentration of the actual trace mineral within the body tissues and fluids, in order that it falls below or rises above the tolerable limits. In such cases, biochemical lesions can develop, physiological functions could also be adversely affected and structural disorders may arise, counting on the dietary deficiency or toxicity of the trace mineral, and therefore the age, sex, or species of the animal.

Trace minerals act as catalysts in enzyme reactions within the cells. Deficiencies and or imbalances of trace minerals can alter the activity of certain enzymes and performance of specific organs thus impairing specific metabolic pathways also as overall immune function. The interactions between trace minerals, system, and disease resistance are extremely complex. Trace minerals play a crucial role within the immune reaction. Inadequate trace mineral nutrition may be a risk factor for poor health.Of the trace minerals, selenium plays a major role, but copper, zinc and iodine deficiencies can also affect the animal health. The animal must be supplied with a diet which contains the required minerals, vitamins, as well as other nutrients, in adequate amounts, proper proportions, and available forms. In trace mineral deficiencies, toxicities, and imbalances, immune function can be suppressed and the production and health of the animal may be affected. Metals weaken the immune system and cause increased vulnerability to viral, bacterial, fungal, and parasitic infections. In an effort to detoxify these substances, bowels, kidneys, liver, and system are overloaded.

Zinc may be a constituent of several enzymes and proteins. Zinc containing enzymes are involved in carbohydrate, lipid, protein, and macromolecule metabolismIt is important for maintaining the integrity of the epithelium , cellular division and repair, and vitamin A transport and utilization. deficiency disease reduces immune reaction and disease resistance. Zinc deficient lambs had a lower percentage of lymphocytes and a higher percentage of neutrophils in their blood.

Copper is required for a number of enzymes (cytochrome oxidase, lysyl oxidase, superoxide dismutase, dopamine- $\beta$-hydroxylase, tyrosinase, and ceruloplasmin) that are involved in several important body functions including cellular respiration, bone formation, heart function, lipid metabolism and etc. Copper deficiency suppresses cell mediated as well as humoral immunity.

Selenium is required by animals and humans for proper growth and immune function. Selenium functions as a component of glutathione peroxidase. It is required for its mineral functions and also has a major role as an antioxidant in the mopping up of damaging free radicals. Selenium affects specific components of the immune system. Polymorphonuclear leukocyte function was shown to be reduced in goats and cattle fed seleniumdeficient diets compared with controls receiving selenium adequate diets. It influences carbohydrate metabolism, lipid metabolism, and protein absorption and metabolism. 
Chromium is essential for mammals. It influences carbohydrate metabolism, lipid metabolism, and protein absorption and metabolism.

Lead can block red blood cell formation. Potassium ion is required for normal cellular function. Severe potassium deficiency is characterized by hypokalemia. The poor consequences of hypokalemia comprise cardiac arrhythmias, glucose intolerance and muscle weakness. The poor effects of insufficient potassium ingestion can result from a lack of potassium per se, a deficiency of its conjugate anion. Just in case of an inadequate intake of bicarbonate precursors, buffers within the bone matrix neutralize excess acid and within the process bone becomes demineralized Increased bone turnover and calcium-containing kidney stones are the resulting adverse consequences. Sodium and chloride ions are required to take care of ECF volume and serum osmolarity.

Sulfate ion is required by the body for synthesis of 3'-phosphoadenosine-5'-phosphosulfate (PAPS), which successively is employed for synthesis of the many important sulfur-containing compounds like chondroitin sulfate and cerebroside sulphate. While substantial levels of sulfate are found in foods and various sources of beverage, the main source of inorganic sulfate is from body protein turnover of the sulfur. Sulfate to be used in PAPS biosynthesis is provided by the inorganic sulfate in food and water, sulfate derived from methionine and cysteine found in dietary protein, and therefore the cysteine.

\section{The Immune System and Immunity to Diseases}

The immune system recognizes self from non-self and acts against the non - self to prevent diseases and ensure the survival of the individual. The immune system not only removes toxins but also repairs, monitors, and defends the body from foreign disease-causing agents such as the bacteria and viruses. Most degenerative diseases are caused by immune dysfunction. It protects animal health and contributes to well-being of the animal. Nonspecific immunity refers to the essential resistance to syndrome that a species possesses. Innate immunity comprises four types of defensive barriers: 1) anatomic (skin), 2) physiologic (temperature, pH, oxygen tension), 3) phagocytic (ingestion of macromolecules by macrophages and neutrophils), and 4) inflammatory Acquired or specific immunity is induced by exposure to an antigen, naturally or via vaccination. Acquired or specific immunity can be divided into two categories: humoral and cell mediated immunity. The humoral immunity involves the interaction of B cells with extracellular antigen and their subsequent proliferation and differentiation into antibody-secreting cells that are specific for a certain antigen. Antibodies secreted by $\mathrm{B}$ cells function because the effector of the humoral immune reaction by binding to an extracellular antigen and neutralizing and/or facilitating its elimination. The immune system is able to discriminate between foreign molecules and the body's own cells and proteins. It mounts an appropriate response known as the effector response to eliminate a specific type of pathogen and induces immunological memory specific to the pathogen. Subsequent exposure to the same foreign organism activates the pathogen - specific memory and elicits a high magnitude immune response to eliminate the pathogen and prevent disease.

\section{Nutrition and immune function}

Nutrition is an important modulator of immune function. It can alter the balance between health and disease. There are three main types of disease fighting nutrients and immune system enhancers; phytochemicals, antioxidants, and polysaccharides. These are essential to boost, strengthen and balance the immune system. Phytochemicals, antioxidants, and polysaccharides are vital disease-fighting nutrients. Food provides energy and amino acids that are required for the development, maintenance and functioning of the immune system. Substrates are needed for the activity of cells of the immune system such as proliferation and antibody production and secretion of acute proteins by the liver.

In young animals, a severe deficiency of any nutrient can impair immunocompetence. The immune system uses many types of glucose and amino acid transporters. Activated leukocytes express high levels of nutrient transporters to easily obtain necessary nutrients even when they are at low concentrations. The immune system can also obtain nutrients from muscle and other tissues. On stimulation by pathogens, the leukocytes release pro-inflammatory cytokines like interleukin 1, tumor necrosis factor and interleukin. Some trace minerals like iron, copper, and zinc have low concentration in muscles. They are essential for the immune system and its functions. The requirement of some trace minerals may be higher for optimal immune function than for growth or reproductive performance. The optimum requirements of nutrients for the development of the immune system in the young animal, its maintenance during good health, and during a challenge by a pathogen should Nutrients in the diet can directly affect the functions of leukocytes and hence the immune response. Dietary factors can alter the levels of prostaglandins and other eicosanoids released by the leukocytes. Membrane fatty acids are the precursors of eicosanoids.

The potency and properties of the eicosanoids released during immune responses change with the composition of 
dietary fat. Dietary nutrients can affect the communication and regulatory decisions made by cells in the immune system. Lipids, vitamins A, D, and E, xanthophylls, some amino acids and bioactive minerals have regulatory actions. Diet influences the hormones that modulate the functioning of the immune system. Diet profoundly affects insulin, glucagon, glucocorticoid, and IGF levels, which can change the type and duration of the immune response. Chronic severe feed restriction results in elevated levels of glucocorticoids, which depress T-cell function and decrease immunocompetence. Protein to calorie ratios and presenting food ad libitum instead of a few large daily meals can also influence immunity through their effects on hormone levels. Feeds containing non-nutrient substances affect immunocompetency through the influence on the leukocyte function, the integrity of the intestinal epithelia, or the population of commensal microorganisms in the intestines. An optimal immune response can occur only under appropriate conditions. Immune responses with the wrong type of leukocyte populations or underresponsiveness can increase the incidence of infectious diseases, on the other hand, exaggerated responses can result in a variety Animals in production can develop anorexia, impaired growth, and other systemic stress responses.

Poor nutrition causes poor immunocompetency which can result in greater incidence and duration of infections, which cause decreased food intake, nutrient losses, and impaired animal health. Vitamin supplementation, especially $\mathrm{E}$ and $\mathrm{A}$, assists the immune system by preventing deficencies. Vitamin A is important because it keeps the epithelial tissues and mucous membrane barriers intact and help stop pathogens. It also aids the immune system by keeping the thymus and bursa of Fabricius functioning, since birds with a vitamin A deficiency have fewer circulating lymphocytes. Vitamin A modulates lymphocyte response to protect the body from diseases. It is an antioxidant and prevents oxidation of phospholipids (mainly polyunsaturated fatty acids) in the cell membrane. In the absence of vitamin $\mathrm{E}$ or other antioxidants, phospholipids oxidize and form prostaglandins, which inhibit inflammation and suppress immune response.

\section{Conclusion}

Adequate and balanced nutrition is vital for optimum development and functioning of the immune system. Absences or deficiencies of various nutrients can impair the immune responses leading to diseases and even death.

\section{Reference}

[1] Abraham, A. B., B. A. Brooks, and U. Eylath. 1991. Chromium and cholesterol-induced arteriosclerosis in rabbits. Ann. Nutr. Metab. 35:203-207.

[2] Azizi, E. S., P. H. Klesius, and J. C. Frandsen. 1984. Effects of selenium on

[3] polymorphonuclear leukocyte function in goats. Am. J. Vet. Res. 45:1715-1719.

[4] Chesters, J. K. 1997. Zinc. Handbook of Nutritionally Essential Mineral Elements. Eds. B. L. O'Dell and R. A. Sunde. Marcel Dekker Inc. New York, New York.

[5] Cook, M.E. 1991. Nutrition and the Immune Response of the Domestic Fowl. Cr. Rev. Poult. Biol. 3:167-190.

[6] Fritsche, K.L., M. Byrge, and C. Feng. 1999. Dietary omega-3 polyunsaturated fatty acids from fish oil reduce interleukin-12 and interferon-gamma production in mice. Immunol. Let. 65:167-173.

[7] Galyean, M. L., L. J. Perino, and G. C. Duff. 1999. Interaction of cattle health/immunity and nutrition. J. Anim. Sci. 77:1120-1134

[8] Gyang, E. O., J. B. Stevens, W. G. Olsen, S. D. Tsitsamis, and E. A. Usenik. 1984. Effects of selenium-vitamin E injection on bovine polymorponucleated leukocyte phagocytosis and killing of Staphylococcus aureus. Am. J. Vet. Res. 45:175-183.

[9] Kaenko, J. J. 1989. Clinical Biochemistry of Domestic Animals. Academic Press Inc. San Diego, CA.

[10] Kornegay, E. T., Z. Wang, C. M. Wood, and N. D. Lindemann. 1997. Supplemental

[11] chromium picolinate influences nitrogen balance, dry matter digestibility, and carcass

[12] traits in growing and finishing pigs. J. Anim. Sci. 75:1319-1323.

[13] Kuby, J. 1994. Immunology. 2nd Ed. W. H. Freeman and Company. New York.

[14] McDowell, L. R. 1989. Vitamins in Animal Nutrition. Academic Press Inc. Harcourt Brace Jovanovich Publishers, San Diego, CA.

[15] McDowell, L. R. 1992. Minerals in Animal and Human Nutrition. Academic Press Inc. Harcourt Brace Jovanovich Publishers, San Diego, CA.

[16] Mertz, W. 1993. Chromium in human nutrition: A review. J. Nutr. 123:626-633

[17] Mulhern, S. A., G. L. Taylor, L. E. Magruder, and A. R. Vessey. 1985. Deficient levels of dietary selenium suppress the antibody response in first and second generation mice. Nutr. Res. 5:201-209.

[18] Okada, S., M. Suzuki, and H. Ohba. 1983. Enhancement of ribonucleic acids synthesis by chromium (III) in mouse liver. J. Inorg. Biochem. 19:95-103.

[19] Schwarz, K. and W. Mertz. 1959. Chromium (III) and the glucose tolerance factor. Archives of Biochemistry and Biophysics. 85:292295.

[20] Underwood, E. J. 1971. Trace Elements in Human and Animal Nutrition 3rd Ed. Academic Press. New York, New York.

[21] Underwood, E. J., and N. F. Suttle. 1999. In: The Mineral Nutrition of Livestock 3rd Ed. CABI Publishing, CAB International, Wallingford, Oxon, UK. 